\title{
POISSON APPROXIMATION FOR SUMS OF INDEPENDENT BIVARIATE BERNOULLI VECTORS
}

\author{
By Mieczyseaw POLAK
}

1. Introduction. It is well known fact as Poisson's theorem that for a given sequence of $\left\{p_{n}, n \geqq 1\right\}$ such that $p_{n} \rightarrow 0 \quad(n \rightarrow \infty)$ we have

$$
P_{n}(m)-\left(\lambda_{n}^{m} / m !\right) e^{-\lambda_{n}} \longrightarrow 0 \quad \text { as } n \rightarrow \infty
$$

for all non-negative integer $m$ where

$$
\lambda_{n}=n p_{n}, \quad P_{n}(m)=\left(\begin{array}{c}
n \\
m
\end{array}\right) p_{\mathfrak{k}}^{m}(1-n)^{n-m} .
$$

Furthermore, if $n p_{n} \rightarrow \lambda(n \rightarrow \infty)$ then we have

$$
P_{n}(m) \longrightarrow\left(\lambda^{m} / m !\right) e^{-\lambda} \quad \text { as } n \rightarrow \infty \text {. }
$$

R. von Mises in the paper [3] has showed that if $\left\{X_{k \jmath}, k \geqq 1, j=1,2, \cdots, n_{k}\right\}$ is a sequence of independent random variables such that

and

$$
P\left[X_{k \jmath}=1\right]=1-P\left[X_{k \jmath}=0\right]=p_{k \jmath}, \quad \jmath=1,2, \cdots, n_{k}
$$

$$
\max _{1 \leq j \leq n_{k}} p_{k \jmath} \longrightarrow 0, \quad \sum_{\jmath=1}^{n_{k}} p_{k \jmath} \longrightarrow \lambda>0 \quad(k \rightarrow \infty),
$$

then

$$
P\left[\sum_{j=1}^{n_{k}} X_{k \jmath}=m\right] \longrightarrow\left(\lambda^{m} / m !\right) e^{-\lambda} \quad(k \rightarrow \infty) .
$$

In (1977) J. Mačys (see. [2]) has proved that the conditions (1.1) are necessary as well.

Let $\left\{\left(X_{k}, Y_{k}\right), k \geqq 1\right\}$ be a sequence of random vectors bivariate Bernoulli law, i.e.

$$
\begin{array}{ll}
P\left[X_{k}=0, Y_{k}=0\right]=p_{00}, & P\left[X_{k}=1, Y_{k}=0\right]=p_{10}, \\
P\left[X_{k}=0, Y_{k}=1\right]=p_{01}, & P\left[X_{k}=1, Y_{k}=1\right]=p_{11},
\end{array}
$$

where $p_{00}+p_{10}+p_{01}+p_{11}=1$.

Received July 30, 1981 
K. Kawamura in [1] has proved that if $\left\{\left(X_{k}, Y_{k}\right), k \geqq 1\right\}$ are mutually independent and indentically distributed random vectors having bivariate Bernoulli probability, then

$$
P\left[\sum_{k=1}^{n}\left(X_{k}, Y_{k}\right)=(n, m)\right] \rightarrow \sum_{s=0}^{\min (n, m)} \frac{\lambda_{10}^{n-s} \lambda_{01}^{m-s} \lambda_{11}^{s}}{(n-s) !(m-s) ! s !} e^{-\left(\lambda_{10}+\lambda_{01}+\lambda_{11}\right)}
$$

as $n \rightarrow \infty$, where $n p_{11}=\lambda_{11}, n p_{10}=\lambda_{10}$ and $n p_{01}=\lambda_{01}$ are fixed values.

The main aim of this paper is to generalize Kawamura's results [1] to nonidentically distributed random vectors $\left\{\left(X_{k}, Y_{k}\right), k \geqq 1\right\}$. The results presented in Section 2 extend those of Kawamura [1], and Mačys [2].

\section{The result.}

Let $\left\{\left(X_{k \jmath}, Y_{k j}\right), \jmath=1,2, \cdots, n_{k}, k \geqq 1\right\}$ be a sequence of independent bivariate Bernoulli vectors with

$$
\begin{array}{ll}
P\left[X_{k \jmath}=0, Y_{k \jmath}=0\right]=p_{k \jmath}(0,0), & P\left[X_{k \jmath}=0, Y_{k \jmath}=1\right]=p_{k j}(0,1), \\
P\left[X_{k \jmath}=1, Y_{k \jmath}=0\right]=p_{k j}(1,0), & P\left[X_{k \jmath}=1, Y_{k \jmath}=1\right]=p_{k \jmath}(1,1),
\end{array}
$$

where $p_{k j}(0,0)+p_{k j}(0,1)+p_{k j}(1,0)+p_{k j}(1,1)=1$.

Let

$$
S_{k}=\sum_{j=1}^{n_{k}}\left(X_{k \jmath}, Y_{k j}\right), \quad k \geqq 1
$$

THEOREM. In order that

$$
\lim _{k \rightarrow \infty} P\left[S_{k}=(n, m)\right]=\sum_{s=0}^{m i n} \frac{\lambda_{10}^{n-s} \lambda_{01}^{m-s} \lambda_{11}^{s}}{(n-s) !(m-s) ! s !} e^{-\left(\lambda_{10}+\lambda_{01}+\lambda_{11}\right)}
$$

may hold for all $n, m \geqq 0$ it is necessary and sufficient that for $k \rightarrow \infty$

$$
\begin{aligned}
& \sum_{j=1}^{n_{k}} p_{k j}(1,0) \longrightarrow \lambda_{10}, \\
& \sum_{j=1}^{n_{k}} p_{k j}(0,1) \longrightarrow \lambda_{01}, \\
& \sum_{j=1}^{n_{k}} p_{k j}(1,1) \longrightarrow \lambda_{11}
\end{aligned}
$$

and

$$
\min _{1 \leq k \leqq n_{k}} p_{k j}(0,0) \longrightarrow 1
$$

Proof. For the sake of simplicity the index $k$ will be omitted in the proof of Theorem, i. e. instead of $p_{k j}$ we write $p_{\jmath}$.

The part if. It is easy to see that 


$$
\begin{aligned}
P\left[S_{k}=(0,0)\right] & =\prod_{g=1}^{n_{k}} p_{g}(0,0), \\
P\left[S_{k}=(1,0)\right] & =\sum_{t=1}^{n_{k}}\left\{p_{t}(1,0) \prod_{\substack{g=1 \\
\neq=1}}^{n_{k}} p_{g}(0,0)\right\} \\
& =\prod_{g=1}^{n_{k}} p_{g}(0,0) \sum_{t=1}^{n_{k}} p_{t}(1,0) / p_{t}(0,0), \\
P\left[S_{k}=(2,0)\right] & =\sum_{t_{1}<t_{2}}^{n_{k}}\left\{p_{t_{1}}(1,0) p_{t_{2}}(1,0) \prod_{\substack{g=1 \\
\neq t_{1}, t_{2}}}^{n_{k}} p_{g}(0,0)\right\} \\
& =\prod_{g=1}^{n_{k}} p_{g}(0,0) \sum_{t_{1}<t_{2}} p_{t_{1}}(1,0) p_{t_{2}}(1,0) / p_{t_{1}}(0,0) p_{t_{2}}(0,0) .
\end{aligned}
$$

In the same way, for every $n>2$, we obtain

$$
\begin{aligned}
P\left[S_{k}=(n, 0)\right] & =\sum_{t_{1}<\cdots<t_{n}}\left\{\prod_{l=1}^{n} p_{t_{l}}(1,0) \prod_{\substack{g=1 \\
\neq t_{1}, \cdots, t_{n}}}^{n_{k}} p_{g}(0,0)\right\} \\
& =\prod_{g=1}^{n_{k}} p_{g}(0,0) \sum_{t_{1}<\cdots<t_{n}}\left(\prod_{l=1}^{n} p_{t_{l}}(1,0) / p_{t_{l}}(0,0)\right) .
\end{aligned}
$$

Let $n, m \geqq 1$ be given and assume that $n=m$. If we put $\delta=\max \left\{2 n-n_{k}, 0\right\}$, then

$$
\begin{aligned}
& P\left[S_{k}=(n, n)\right]=\sum_{l_{1}<\cdots<i_{n} \jmath_{1}<\cdots<j_{n}} P\left[\bigcap_{p=1}^{n}\left[X_{j_{p}}=1\right], \bigcap_{\substack{l=1 \\
\neq l_{1}, \cdots, \nu_{n}}}^{n_{k}}\left[X_{l}=0\right],\right. \\
& \left.\cdot \bigcap_{r=1}^{n}\left[Y_{J_{r}}=1\right], \bigcap_{\substack{s_{j=1} \\
\neq j_{1}, \cdots, j_{n}}}^{n_{k}}\left[Y_{s}=0\right]\right]=\sum_{i_{1}<\cdots<i_{n}}\left\{\prod_{p=1}^{n} p_{\imath_{p}}(1,1) \prod_{\substack{g=1 \\
\neq i_{1}, \cdots, i_{n}}}^{n_{k}} p_{g}(0,0)\right\}
\end{aligned}
$$

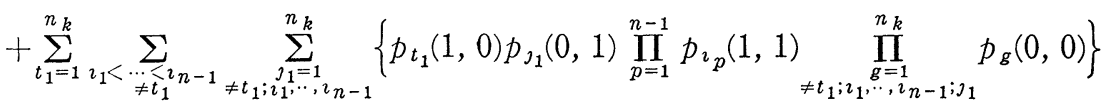

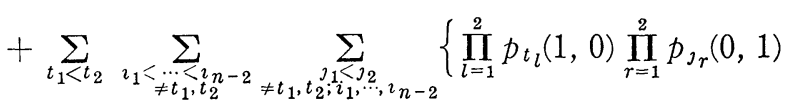

$$
\begin{aligned}
& \left.\cdot \prod_{p=1}^{n-2} p_{\imath_{p}}(1,1) \prod_{\substack{g=1 \\
\neq t_{1} t_{2} ; \imath_{1}, \cdots, \imath_{n-2} ; J_{1}, \jmath_{2}}}^{n_{k}} p_{g}(0,0)\right\}+\cdots+\sum_{t_{1}<\cdots<t_{n}-\delta} \sum_{\imath_{1}<\cdots<i \delta}
\end{aligned}
$$

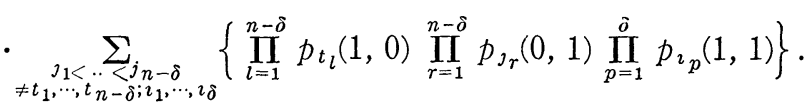

Taking out the product $\prod_{g=1}^{n_{k}} p_{g}(0,0)$ before the sign of the first sum, we may write out

(2.8) $\quad P\left[S_{k}=(n, n)\right]=\sum_{\left.s=\max \mid 2 n-n_{k}, 0\right\}}^{n} \prod_{g=1}^{n_{k}} p_{g}(0,0)\left\{\sum_{t_{1}<\cdots<t_{n-s}}\left(\prod_{l=1}^{n-s} p_{t_{l}}(1,0) / p_{t_{l}}(0,0)\right.\right.$ 


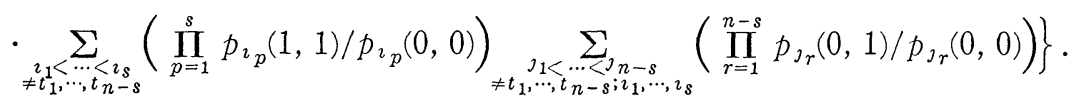

Let now $n, m \geqq 1$ be given and suppose that $n<m$. If we put $\delta=$ $\max \left\{n+m-n_{k}, 0\right\}$, then

$$
\begin{aligned}
& P\left[S_{k}=(n, m)\right]=\sum_{\imath_{1}<\cdots<i_{n} \jmath_{1}<\cdots<j_{m}} P\left[\bigcap_{p=1}^{n}\left[X_{\imath_{p}}=1\right], \bigcap_{\substack{l=1 \\
\neq \imath_{1}, \cdots, \imath_{n}}}^{n_{k}}\left[X_{l}=0\right], \bigcap_{r=1}^{m}\left[Y_{J_{r}}=1\right],\right.
\end{aligned}
$$

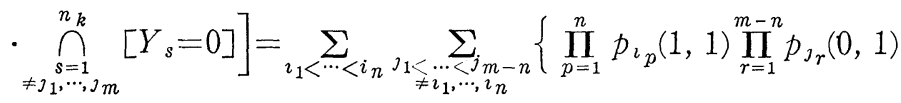

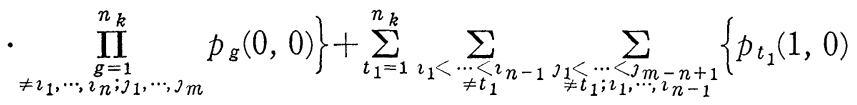

$$
\begin{aligned}
& \left.\cdot \prod_{r=1}^{m-n+1} p_{J_{r}}(0,1) \prod_{p=1}^{n-1} p_{\imath_{p}}(1,1) \underset{\substack{g=1 \\
\neq t_{1} ; \imath_{1}, \cdots, \imath_{n}-1 ; j_{1}, \cdots, \jmath_{m-n+1}}}{n_{k}} p_{g}(0,0)\right\}
\end{aligned}
$$

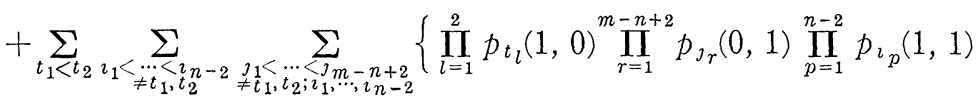

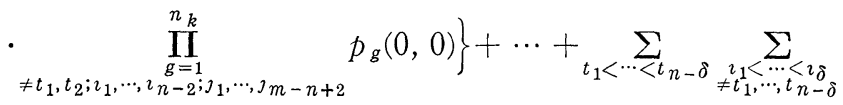

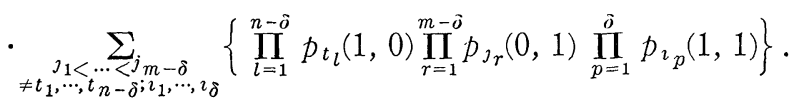

Thus taking into account (2.8) and the above given equality, for all $n, m \geqq 1$, we may write

$$
\begin{aligned}
& P\left[S_{k}=(n, m)\right]=\sum_{s=\max \left(n+m-n_{k}, 0\right\}}^{\min (n, m)} \prod_{g=1}^{n_{k}}\left\{p_{g}(0,0) \sum_{t_{1}<\cdots<t_{n-s}}\left(\prod_{l=1}^{n-s} p_{t_{l}}(1,0) / p_{t_{l}}(0,0)\right)\right. \\
& \left.\cdot \sum_{\substack{\imath_{1}<\cdots<i_{s} \\
\neq t_{1}, \cdots, t_{n}-s}}\left(\prod_{p=1}^{s} p_{\imath_{p}}(1,1) / p_{\imath_{p}}(0,0)\right) \underset{\substack{j_{1}<\ldots<j_{m-s} \\
\neq t_{1}, \cdots, t_{n-s} ; i_{1}, \cdots, \imath_{s}}}{\sum_{r=1}}\left(\prod_{r=1}^{m-s} p_{J_{r}}(0,1) / p_{J_{r}}(0,0)\right)\right\} .
\end{aligned}
$$

In order to prove that

$$
\prod_{g=1}^{n_{k}} p_{g}(0,0) \longrightarrow e^{-\left(\lambda_{10}+\lambda_{01}+\lambda_{11}\right)} \quad \text { as } \quad k \rightarrow \infty,
$$

we consider the inequality $1+y \leqq e^{y}, \quad y \in[-1, \infty)$. Putting $y=-x, \quad x \in[0,1)$, we have

$$
e^{-x /(1-x)} \leqq 1-x \leqq e^{-x}, \quad x \in[0,1) .
$$

Now putting $\Lambda_{g}=p_{g}(1,0)+p_{g}(0,1)+p_{g}(1,1)$ and using the last inequality, we obtain

$$
e^{-\frac{1}{\min p_{g}(0,0)}}{ }_{g=1}^{n_{k}} A_{g} \leqq \prod_{g=1}^{n_{k}} p_{g}(0,0) \leqq e^{-\Sigma A_{g}}
$$


Taking into account (2.1)-(2.4) one can prove that

$$
\lim _{k \rightarrow \infty} \prod_{g=1}^{n_{k}} p_{g}(0,0)=e^{-\left(\lambda_{10}+\lambda_{01}+\lambda_{11}\right)}
$$

Now we are going to prove that

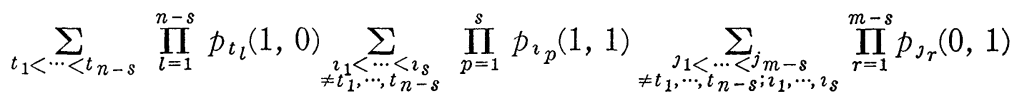

$$
\begin{aligned}
& \longrightarrow \lambda_{10}^{n-s} /(n-s) ! \cdot \lambda_{11}^{s} / s ! \cdot \lambda_{01}^{m-s} /(m-s) ! \quad \text { as } \quad k \rightarrow \infty .
\end{aligned}
$$

In the case $m=0$ the proof is by induction with respect to $n$. The case $n=1$ is obvious. Let $n=2$, then by (2.1) and (2.4)

$$
\sum_{t_{1}<t_{2}} p_{t_{1}}(1,0) p_{t_{2}}(1,0) \longrightarrow \lambda_{10}^{2} / 2
$$

as

$$
2 \sum_{t_{1}<t_{2}} p_{t_{1}}(1,0) p_{t_{2}}(1,0)=\left(\sum_{t=1}^{n_{k}} p_{t}(1,0)\right)^{2}-\sum_{t=1}^{n_{k}} p_{t}^{2}(1,0)
$$

and

$$
0 \leqq \sum_{t=1}^{n_{k}} p_{t}^{2}(1,0) \leqq\left(1-\min p_{t}(0,0)\right) \cdot \sum_{t=1}^{n_{k}} p_{t}(1,0) .
$$

Assume that

$$
\sum_{t_{1}<\cdots<t_{n-1}} \prod_{l=1}^{n-1} p_{t_{l}}(1,0) \longrightarrow \lambda_{10}^{n-1} /(n-1) !
$$

Multiplying the left hand side of the last relation by $\sum_{t_{n}=1}^{n_{k}} p_{t_{n}}(1,0)$ we obtain

$$
\begin{aligned}
& \sum_{t_{1}<\cdots<t_{n-1}} p_{t_{1}}^{2}(1,0) \prod_{\substack{l=1 \\
\neq t_{1}}}^{n-1} p_{t_{l}}(1,0)+\sum_{t_{1}<\cdots<t_{n-1}} p_{t_{2}}^{2}(1,0) \prod_{\substack{l=1 \\
\neq t_{2}}}^{n-1} p_{t_{l}}(1,0)+\cdots \\
& \quad+\sum_{t_{1}<\cdots<t_{n-1}} p_{t_{n-1}}^{2}(1,0) \prod_{\substack{l=1 \\
\neq t_{n-1}}}^{n-1} p_{t_{l}}(1,0)+\sum_{t_{n}<t_{1}<\cdots<t_{n-1}} \prod_{l=1}^{n} p_{t_{l}}(1,0) \\
& \quad+\sum_{t_{1}<t_{n}<\cdots<t_{n-1}} \prod_{l=1}^{n} p_{t_{l}}(1,0)+\cdots+\sum_{t_{1}<\cdots<t_{n-1}<t_{n}} \prod_{l=1}^{n} n_{t_{l}}(1,0) .
\end{aligned}
$$

Because every sum from among the first $(n-1)$ sums we may estimate by

$$
\left(1-\min p_{t}(0,0)\right) \sum_{t_{1}<\cdots<t_{n-1}} \prod_{l=1}^{n-1} p_{t_{l}}(1,0)
$$

so the first $(n-1)$ sums tend to 0 . We have then

$$
n \sum_{t_{1}<\cdots<t_{n}} \prod_{l=1}^{n} p_{t_{l}}(1,0) \longrightarrow \lambda_{10}^{n} /(n-1) !
$$

The last relation proves that 


$$
\sum_{t_{1}<\cdots<t_{n}} \prod_{l=1}^{n} p_{t_{l}}(1,0) \longrightarrow \lambda_{10}^{n} / n ! .
$$

In the same way one can prove that

$$
\sum_{\imath_{1}<\cdots<i_{s}} \prod_{p=1}^{s} p_{\imath_{p}}(1,1) \longrightarrow \lambda_{11}^{s} / s !
$$

and

$$
\sum_{\jmath_{1}<\cdots<s_{m-s}} \prod_{r=1}^{m-s} p_{\jmath_{r}}(0,1) \longrightarrow \lambda_{01}^{m-s} /(m-s) ! .
$$

Let us put

$$
\begin{aligned}
B_{n_{k}}(n-s, s, m-s)= & \sum_{t_{1}<\cdots<t_{n-s}} \prod_{l=1}^{n-s} p_{t_{l}}(1,0) \sum_{i_{1}<\cdots<i_{s}} \prod_{p=1}^{s} p_{\imath_{p}}(1,1) \\
& \cdot \sum_{\partial_{1}<\cdots<s_{m-s}} \prod_{r=1}^{m-s} p_{J_{r}}(0,1) .
\end{aligned}
$$

Then, taking into account (2.11)-(2.13), we have

$$
B_{n_{k}}(n-s, s, m-s) \longrightarrow \lambda_{10}^{n}-s /(n-s) ! \cdot \lambda_{11}^{s} / s ! \cdot \lambda_{01}^{m-s} /(m-s) !
$$

as $k \rightarrow \infty$.

Let us define

and

$$
\underset{t_{1}, \cdots, t_{n-s}}{A^{s}}(1,1)=\sum_{\imath_{1}<\cdots<i_{s}} \prod_{p=1}^{s} p_{\imath_{p}}(1,1)-\sum_{\substack{i_{1}<\cdots<i_{s} \\ \neq t_{1}, \cdots, t_{n-s}}} \prod_{p=1}^{s} p_{\imath_{p}}(1,1)
$$

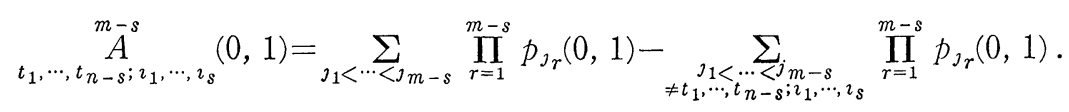

Taking into account the above considerations it may be proved that $\underset{t_{1}, \cdots, t_{n-s}}{A s}(1,1)$ and $\underset{t_{1}, \cdots t_{n-s} ; \imath_{1}, \cdots, v_{s}}{A}(0,1)$ tend to 0 as $k \rightarrow \infty$.

It is easy to see that

where

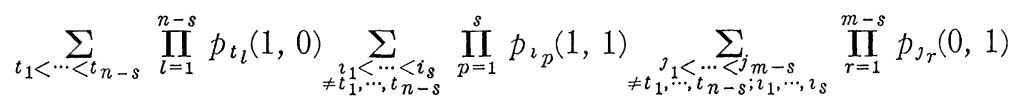

$$
\begin{aligned}
& =B_{n_{k}}(n-s, s, m-s)-\left(Z_{1}+Z_{2}+Z_{3}\right) \text {, }
\end{aligned}
$$

$$
\begin{aligned}
& Z_{1}=\sum_{t_{1}<\cdots<t_{n-s}} \prod_{l=1}^{n-s} p_{t_{l}}(1,0) \underset{t_{1}, \cdots, t_{n-s}}{\stackrel{n-s}{A}}(0,1) \sum_{j_{1}<\cdots<j_{m-s}} \prod_{r=1}^{m-s} p_{J_{r}}(0,1), \\
& Z_{2}=\sum_{t_{1}<\cdots<t_{n-s}} \prod_{l=1}^{n-s} p_{t_{l}}(1,0) \sum_{i_{1}<\cdots<i_{s}} \prod_{p=1}^{s} p_{\imath_{p}}(1,0){ }_{t_{1}, \cdots, t_{n-s} ; \imath_{1}, \cdots, t_{s}}^{m-s}(0,1),
\end{aligned}
$$




$$
Z_{3}=\sum_{t_{1}<\cdots<t_{n-s}} \prod_{l=1}^{n-s} p_{t_{l}}(1,0) \underset{t_{1}, \cdots, t_{n-s}}{A}(1,1) \underset{t_{1}, \cdots, t_{n-s} ; i_{1}, \cdots, t_{s}}{A}(0,1)
$$

and $Z_{i} \rightarrow 0$ as $k \rightarrow \infty, 1 \leqq \imath \leqq 3$.

The relation (2.10) finishes the proof of the part if, because

$$
\begin{aligned}
& \sum_{t_{1}<\cdots<t_{n-s}} \prod_{l=1}^{n-s} p_{t_{l}}(1,0) \sum_{\substack{t_{1}<\cdots, i_{s} \\
\neq t_{1}, \cdots, t_{n-s}}} \prod_{p=1}^{s} p_{i_{p}}(1,1) \sum_{\substack{j_{1} \\
\neq t_{1}, \cdots, t_{n-j}<j_{m-1}, \cdots, l_{s}}} \prod_{r=1}^{m-s} p_{J_{r}}(0,1) \\
& \leqq \sum_{t_{1}<\cdots<t_{n-s}} \prod_{l=1}^{n-s} p_{t_{l}}(1,0) / p_{t_{l}}(0,0) \sum_{\substack{\tau_{1}, \cdots, i_{s} \\
\neq t_{1}, \cdots, t_{n-s}}} \prod_{p=1}^{s} p_{\imath_{p}}(1,1) / p_{\imath_{p}}(0,0) / \\
& \underset{\substack{{ }_{1}<\cdots<j_{m}-s \\
\neq t_{1}, \cdots, t_{n}-s ; i_{1}, \cdots, \imath_{s}}}{\sum} \prod_{r=1}^{m-s} p_{J_{r}}(0,1) / p_{J_{r}}(0,0) \\
& \leqq\left(\frac{1}{\min p_{j}(0,0)}\right)^{n+m-s} \cdot \sum_{t_{1}<\cdots<t_{n-s}} \prod_{l=1}^{n-s} p_{t_{l}}(1,0)
\end{aligned}
$$

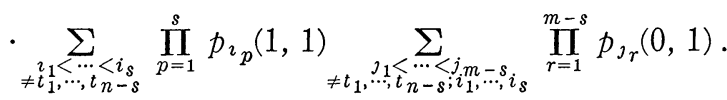

Proof of the part only. From (2.5)-(2.7) we have

$$
\begin{gathered}
\prod_{g=1}^{n_{k}} p_{g}(0,0) \longrightarrow e^{-\left(\lambda_{10}+\lambda_{01}+\lambda_{11}\right)}, \\
\sum_{t_{1}=1}^{n_{k}} p_{t_{1}}(1,0) / p_{t_{1}}(0,0) \longrightarrow \lambda_{10} \\
\sum_{J_{1}=1}^{n_{k}} p_{J_{1}}(0,1) / p_{J_{1}}(0,0) \longrightarrow \lambda_{01}
\end{gathered}
$$

and

$$
\begin{gathered}
\sum_{t_{1}<t_{2}} p_{t_{1}}(1,0) p_{t_{2}}(1,0) / p_{t_{1}}(0,0) p_{t_{2}}(0,0) \longrightarrow \lambda_{10}^{2} / 2 . \\
\sum_{J_{1}<j_{2}} p_{\jmath_{1}}(0,1) p_{\jmath_{2}}(0,1) / p_{J_{1}}(0,0) p_{\jmath_{2}}(0,0) \longrightarrow \lambda_{01}^{2} / 2 .
\end{gathered}
$$

From (2.15)-(2.17) we get

$$
\sum_{t_{1}=1}^{n_{k}}\left(p_{t_{1}}(1,0) / p_{t_{1}}(0,0)\right)^{2} \longrightarrow 0, \quad \sum_{\jmath_{1}=1}^{n_{k}}\left(p_{\jmath_{1}}(0,1) / p_{\jmath_{1}}(0,0)\right)^{2} \longrightarrow 0,
$$

which implies that

$$
\max \left(p_{t}(1,0) / p_{t}(0,0)\right) \longrightarrow 0, \quad \max \left(p_{j}(0,1) / p_{j}(0,0)\right) \longrightarrow 0 .
$$

Now we will prove that $\max \left(p_{i}(1,1) / p_{i}(0,0)\right) \rightarrow 0$. Indeed, from (2.8) for $n=m=1$ and (2.15) we have 


$$
\begin{gathered}
\sum_{t_{1}=1}^{n_{k}} p_{t_{1}}(1,0) / p_{t_{1}}(0,0) \sum_{j_{1}=1}^{n_{k}} p_{\jmath_{1}}(0,1) / p_{j_{1}}(0,0)+\sum_{\imath_{1}=1}^{n_{k}} p_{\imath_{1}}(1,1) / p_{\imath_{1}}(0,0) \\
\longrightarrow \lambda_{10} \cdot \lambda_{01}+\lambda^{11} .
\end{gathered}
$$

On the other hand, taking into account the inequality

$$
\sum_{t_{1}=1}^{n_{k}} p_{t_{1}}(1,0) p_{t_{1}}(0,1) / p_{t_{1}}^{2}(0,0) \leqq \max \left(p_{\jmath}(0,1) / p_{\jmath}(0,0)\right) \sum_{t_{1}=1}^{n_{k}} p_{t_{1}}(1,0) / p_{t_{1}}(0,0),
$$

(2.15) and (2.16), we get

$$
\sum_{\imath_{1}=1}^{n_{k}} p_{\imath_{1}}(1,1) / p_{\imath_{1}}(0,0) \longrightarrow \lambda_{11} .
$$

In the same way, putting in (2.8) $n=m=2$, we obtain

$$
\sum_{i_{1}<i_{2}} p_{\imath_{1}}(1,1) p_{\imath_{2}}(1,1) / p_{\imath_{1}}(0,0) p_{\imath_{2}}(0,0) \longrightarrow \lambda_{11}^{2} / 2 \text {. }
$$

From (2.18) and (2.19) it follows that $\max \left(p_{i}(1,1) / p_{i}(0,0)\right) \rightarrow 0$, and therefore $\min p_{g}(0,0) \rightarrow 1$ as $k \rightarrow \infty$.

The last relation and (2.16) imply that

$$
\sum_{t=1}^{n_{k}} p_{t}(1,0) \longrightarrow \lambda_{10}
$$

because

$$
\min p_{t}(0,0) \sum_{t_{1}=1}^{n_{k}} p_{t_{1}}(1,0) / p_{t_{1}}(0,0) \leqq \sum_{t_{1}=1}^{n_{k}} p_{t_{1}}(1,0) \leqq \sum_{t_{1}=1}^{n_{k}} p_{t_{1}}(1,0) / p_{t_{1}}(0,0) .
$$

In the same way one can prove that (2.2) and (2.3) are satisfied. Thus the proof of Theorem is completed.

\section{REFERENCES}

[1] Kawamura, K., The structure of bivarıate Poisson distributıon. Kōdai Math. Sem. Rep. 25 (1973), 246-256.

[2] Иагис, Ю. Ю., Об испытаниях Бернулли с переменными ьероятностями. Lit. mat. rink. XIX, (1974), 145-151.

[3] Mises, R.v., Über die Wahrscheinlichkeit seltener Ereignisse. Z. Angew. Math. Mech. B. 1, (1921), 121-124.

Institute of Mathematics, Maria Curie-Sktodowska University, Plac M. Curie-Skeodowskiej 1 20-031 Lublin, Poland 